\title{
FBG Sensörlerin Yüzey Uygulamalarında Kullanılan Sabitleme Elemanlarının İncelenmesi ve Özgün Bir Tasarımın Geliştirilmesi
}

\author{
${ }^{* 1}$ Hande Eğin, ${ }^{1}$ Zafer Ünal, ${ }^{1,2}$ Ahmet Can Günaydın \\ ${ }^{1}$ Mühendislik Fakültesi, Bursa Uludağ Üniversitesi Makine Mühendisliği Bölümü, Türkiye \\ ${ }^{2}$ Türk Havacılık ve Uzay Sanayii A.Ş., TUSAŞ Uludağ Üniversitesi Ar-Ge Merkezi, Türkiye
}

\section{Özet:}

Fiber Bragg Izgara (Fiber Bragg Grating (FBG)) sensörler küçük olması, hafif olması, yüksek hassasiyete sahip olması ve elektromanyetik girişimlerden etkilenmemesi gibi avantajları nedeniyle son yıllarda yoğun olarak kullanılmaya ve geliştirilmeye devam etmektedir. Doğrudan gerinim ve sıcaklık; dolaylı olarak ise basınç, yer değiştirme, titreşim ve ivme gibi fiziksel parametreler ölçülebilmektedir. Gerinim ve sicaklık parametrelerini elde etmek için FBG sensörlerin ölçüm yapılacak malzemeyle bağlantı kurulması gerekmektedir. Bu bağlantı genel olarak iki yöntem kullanılarak sağlanmaktadır. Bunlardan ilki yüzeye sabitlenmesi diğeri ise malzemeye gömülmesidir. Yüzeye sabitleme metotlarından bir tanesi sabitleyici eleman kullanmaktır. Sabitleyici eleman; sensörün kolay montaj edilmesi, dış ortamlardan korunması ve hassasiyetinin artması gibi avantajlar sağlamaktadır. Bu bildiride, farklı FBG sensör sabitleyici elemanlarının ölçüm hassasiyeti, sabitleyici boyutu, malzeme türü, sabitleme yöntemi incelenmiş ve karşılaştııılmıştır. Yeni bir sabitleme elemanının tasarımı yapılmış ve sonlu elemanlar yöntemiyle doğrulanmıştır.

Anahtar Kelimeler: Fiber Bragg Izgara (FBG), Sabitleme Metodları, Sensör Sabitleyici Eleman

\begin{abstract}
:
Fiber Bragg Grating (FBG) sensors continue to be used and developed intensively in recent years due to their advantages such as being small, lightweight, having high sensitivity and not being affected by electromagnetic interference. Directly strain and temperature; indirectly physical parameters such as pressure, displacement, vibration and acceleration can be measured. In order to obtain strain and temperature parameters, FBG sensors must be connected with the material that will be measured. Generally, this connection is provided using two methods. One of these is fixing to the surface, the other is embedding in the material. One of the surface fixing methods is to use fixing element (carrier, mounting kit, packaging, etc.). The fixing element provides some advantages such as easy installation of the sensor, protection from external environments and increased sensitivity. In this study, the measurement accuracy, fixing size, material type and fixing type of different FBG sensor fixing elements are examined and compared. A novel fixing element has been designed and verified by the Finite Element Method (FEM).
\end{abstract}

Keywords: Carrier, Fiber Bragg Grating (FBG), Packaging Methods, The Sensor Fixing Element, Strain Transfer

*Sorumlu yazar: Hande Eğin Adres: Mühendislik Fakültesi, Bursa Uludağ Üniversitesi Makine Mühendisliği Bölümü, 16240, Bursa, TÜRKIYE. E-mail adres: handeegin@gmail.com 


\section{Giriş}

Fiber optikler dielektrik saf bir cam üzerinden 1şığın tam yansıma prensibine göre iletilmesiyle çalışmaktadır. Bir optik fiber genel olarak öz (core), kılıf (cladding) ve kaplama (coating) olmak üzere üç bileşenden oluşmaktadır. Kılıf, fỉbere 1şık kaybının, saçılmaların azaltılması ve mekanik özelliklerin iyileștirilmesine katkı sağlamaktadır. Kaplama ise fiberi fiziksel hasarlardan korumak için kullanılmaktadır. Fiber üzerinde basınç, sıcaklık ve gerilme gibi dış etkenlerden kaynaklanan etkiler, 1şığın iletimi üzerinde 1şık şiddeti, faz kayması, dalgaboyu ve polarizasyon değişimlerine sebebiyet vermektedir. Fiber Optik Sensör'lerin (FOS) çalışma prensibi, bu değişimlerin ölçülerek, fiziksel büyüklüklere dönüştürülmesine dayanmaktadır [1]. En yaygın kullanılan dalga boyu tabanlı sensör fiber Bragg 1zgara sensörüdür (Fiber Bragg Grating (FBG)) [2]. FBG sensörler; sağlık, inşaat, havacılık, denizcilik, ulaşım, petrol endüstrileri ve akıllı sistemlerde geniş kullanım alanları bulmaktadır. Bu sensör ile sıcaklık ve gerilmedeki değişimleri yansıyan 1şığın dalga boyundaki kayma ile tespit etmek mümkün olmaktadır [3]. Sensörün küçük boyutlu olması, hafif olması, elektromanyetik girişimlerden etkilenmemesi, çoklama kabiliyetine (multiplexing capability) sahip olması, yüksek hassasiyete sahip olması ve yüksek tekrarlanabilirliğe sahip olması gibi özelliklerinden dolayı, geleneksel sensörlere göre birçok avantaj sunar [4]. FBG'ler ölçüm yapılacak malzemenin üzerine iki farklı teknik kullanılarak yerleştirilir. Birinci teknikte, FBG'ler yüzeye bir yapıştırıcı, sabitleme elemanı ya da her ikisi birden kullanılarak yerleştirilir. Literatürde FBG uygulamaları için farklı tip sabitleme elemanları ve bu sabitleme elemanlarını, malzeme ile bütünleştirerek gerinim transferini sağlamak için montaj, kaynak ya da yapıştırıcı gibi farklı yöntemler kullanılmıştır. İkinci teknikte ise, FBG'lerin küçük boyutlu ve silindirik yapılı olmasının sağladığı avantajlardan ötürü kompozit gibi yapıların içine gömülebilmesidir [5]. Sensörün kaplaması, yapıştırıcı gibi elemanlar üzerinde oluşan bazı kayma gerilmelerinden ve sönümlemelerden ötürü 1zgara ve yüzey arasında süreksizlik meydana gelir. Bu durum gerinim transferine olumsuz etki etmektedir [6]. Bu nedenle, ölçüm hassasiyetini artırmak için FBG sensörlerde 1zgaranın bulunduğu bölgede kaplama kullanılmaması tercih edilebilir. Ancak, çıplak fiber kesme kuvveti yönünde kırılgandır. İmalat sırasında ve sonrasında kolayca hasar görebilir. Çıplak FBG’lerin zarar görmesini önlemek için bazı koruyucu sabitleme elemanları önerilmiştir [7]. Araştırmacılar, FBG'nin genel gerinim hassasiyetini artırmak için test edilen yapı üzerinde uzun bir bölgedeki sürekli gerinimi kısa 1zgara alanına merkezleştirmek için özel sabitleme yöntemleri ve yapıları geliştirmişlerdir [8].

$\mathrm{Bu}$ çalışmada, literatürdeki birçok farklı FBG sensör sabitleyici elemanların ölçüm hassasiyeti, sabitleyici boyutu, malzeme türü, sabitleme yöntemi incelenmiş ve karşılaştırılmıştır. Ayrıca, enstrümantasyon işleminde kullanılan yapıştırıcıların mekanik özelliklerinin ve yapıştırıcının kalınlığının ölçüm sonuçlarına etkisi sonlu elemanlar yöntemiyle ortaya konmuştur. Son olarak, yeni bir sabitleme elemanının tasarımı üzerinde durulmuştur.

\section{FBG Sabitleme Elemanları}

Gerinim, yapıların izlenmesindeki en önemli fiziksel parametrelerden biridir. Genellikle, FBG sensörler gerinim ve sıcaklığı tespit etmek için yapıştırıcılar kullanılarak ölçüm yapılacak yapıların üzerine doğrudan bağlanırlar veya akıllı yapılar oluşturmak için bazı kompozit malzemelere gömülürler. Doğrudan yapıştırılmış veya gömülmüş çıplak FBG sensörlerin gerinim 
hassasiyeti teorik olarak $1.21 \mathrm{pm} / \mu \varepsilon$ 'dir. Bazı durumlarda, test edilecek gerinim düşük bir seviyededir ve çıplak FBG'nin hassasiyeti, FBG sorgulayıcısının (interrogator) gürültüsü gibi nedenlerle, bu küçük genlikli gerinimin tespiti için uygun olmayabilir. Bu nedenle, daha yüksek hassasiyet ve doğruluğa sahip bir FBG gerinim sensörüne ihtiyaç duyulabilir. Hem çıplak FBG'lerin zarar görmesini önlemek hem de hassasiyeti arttırmak için bazı sabitleme elemanları önerilmiştir [7]. Sabitleme elemanları yüzeye yapıştırıcı kullanılarak, kaynaklanarak ya da vida gibi bir bağlantı elemanı ile uygulanabilir (Şekil 1). Yapıştırıcı ve kaynak kullanılarak uygulanan sabitleme elemanlarının aksine bağlantı elemanı kullanılarak yüzeye uygulanan sabitleme elemanları tekrar kullanılabilmektedir. Ancak, ölçüm yapılmak istenen malzeme yüzeyinde delik, kanal benzeri bağlantı bölgeleri oluşturmak gerekmektedir. Bu bölgeler yapı üzerinde süreksizliklere sebep olarak gerilim birikmelerine yol açmaktadır.
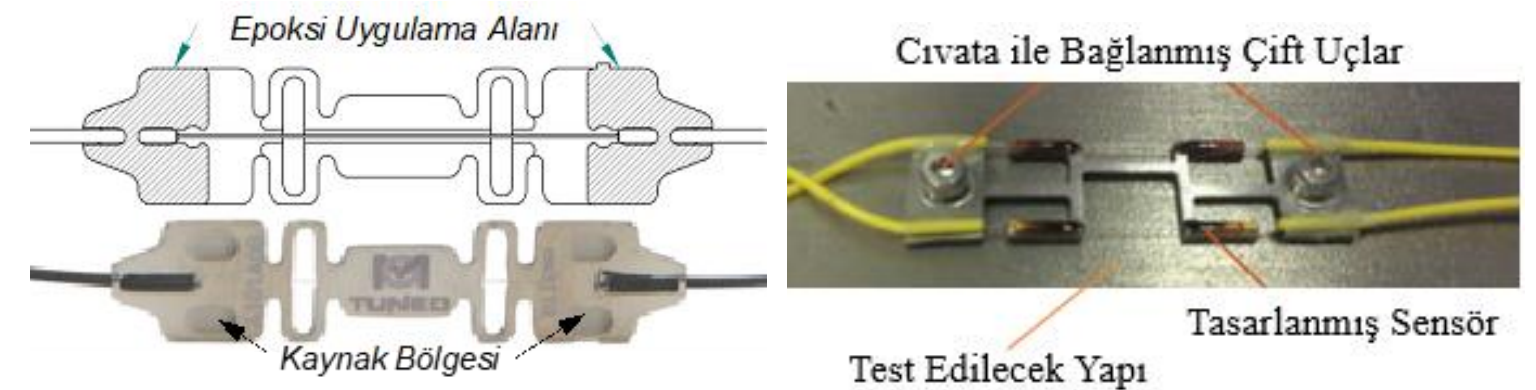

Şekil 1. Sabitleme elemanı örneği $[8,9]$

FBG sensörler için tasarlanan sabitleme elemanları farklı sektörlerde birçok uygulamada kullanılmaktadır. Tasarımına bağlı olarak sağladığı avantajlar ve dezavantajlar değerlendirilerek uygulama için en uygun olan sabitleme elemanının seçilmesi oldukça önemlidir.

Genel olarak 1500-1600 nm dalgaboyu aralığında çalışan FBG sensörlerde yüksek hassasiyet sağlayan sabitleme elemanları kullanılması durumunda gerinimdeki değişim büyük dalga boyu kaymalarına sebep olacaktır. Bu durumda, optik kablo üzerine çok sayıda FBG sensör yerleştirilmesi dalga boylarında girişime sebep olabilir ve verilerin okunmasını imkânsız hale getirebilir. Bu girişimleri engellemek için bir kablo üzerine yerleştirilebilecek FBG sensör sayısı azalacaktır.

Ticari olarak satılan hazır FBG sensörlerde 1zgaranın bulunduğu, ölçüm yapılan, bölgenin boyu genellikle 10-12 mm olmaktadır. Köprülerin yapısal sağlık izlenmesi gibi uygulamalarda FBG sensörlerin yüzeye uygun sabitleyici eleman kullanmadan uygulanması betonun yapısından ötürü doğru sonuçlar vermemektedir. Beton içerisinde kullanılan malzemelerin boyutlarının eşit olmaması ve içerisinde bulundurabileceği boşluklar sebebiyle gerinim dağılımı bölgesel olarak değişkenlik göstermektedir. Bu nedenle büyük boyutlu sabitleyici elemanlar kullanılarak, sabitleyici elemanın bağlantı noktaları arasında kalan bölgenin ortalama gerinimi değerlendirilir.

Yukarıda bahsedilen iki farklı uygulama için örnekler üzerinden inceleme yapıldığında farklı tip sabitleyici elemanlara ihtiyaç duyulduğu görülmektedir. Bu kapsamda FBG sabitleme elemanları üzerine birçok çalışma yapılmıştır. Bazı araştırmacılar, bir FBG'nin iki ucunu sırasıyla iki yüksek sertlikli boru ile birleştirmiş ve daha sonra nispeten daha yüksek bir gerinme hassasiyeti elde etmek için iki borunun dış uçlarını yapı yüzeyine bağlamışlardır. Örneğin, Ren ve diğ., [7] FBG'yi iki tutucu tüp kullanarak paketlemişlerdir ve $2.053 \mathrm{pm} / \mu \varepsilon$ hassasiyet elde edebilen bir FBG 
gerinim sensörü tasarlamışlardır. Li ve diğ. [10], bir otoyol köprüsünün uzun vadeli yapısal sağlık izleme sistemi için aynı şekilde $2.52 \mathrm{pm} / \mu \varepsilon$ hassasiyete sahip bir FBG gerinim sensörü geliştirmişlerdir. Ek olarak, diğer bazı araştırmacılar FBG alanındaki gerinmeyi genişletmek için esnek menteşeli alt tabakalar kullanmışlardır. Zhang ve diğ. [11], sıcaklığa duyarlı olmayan ve gerinme duyarlılığı $1.814 \mathrm{pm} / \mu \varepsilon$ olan elmas çerçeve ile paketlenmiş bir FBG gerinim sensörü sunmuşlardır. Li ve diğ. [12] tasarladıkları sensör ile çıplak bir FBG gerinim sensörünün hassasiyetinin beş katından fazla olan $6.2 \mathrm{pm} / \mu \varepsilon$ hassasiyet elde etmişlerdir. Li ve diğ. [8] daha önce tasarladıkları sensörü geliştirerek iki FBG'nin hassasiyetleri sırasıyla $7.72 \mathrm{pm} / \mu \varepsilon$ ve -2.94 $\mathrm{pm} / \mu \varepsilon$ değerlerine ulaşmıştır. Guo ve diğ. [13], FBG tabanlı bir sensörün gerinim hassasiyetini arttırmak için esnek menteşeli bir alt tabaka kullanmışlardır. Geliştirdikleri sensör ile $3.357 \mathrm{pm} / \mu \varepsilon$ hassasiyete ulaşmışlardır. Bu sensörler, doğrudan yapıştırılan çıplak FBG sensörlerine kıyasla daha yüksek bir gerinme hassasiyeti elde edebilirler. Ancak, bu sensörler ince esnek menteşeler nedeniyle narindir ve kırılması kolaydır. Nawrot ve diğ. [14] FBG'ye uygulanan gerinimi yükseltmek için mekanik bir dönüştürücü önermişlerdir. Bu sensörün dış boyutları 380 x 105 $\mathrm{mm}^{2}$ ' dir. Köprüler, ağır iş tipi portal vinçler gibi büyük mühendislik yapılarında kullanılabilir. Normal boyutlarda endüstriyel ekipmanlarda kullanılmak için çok büyüktür. Yao ve diğ. [15], gerinme, titreşim ve sıcaklığı aynı anda ölçmek için birleşik bir sensör geliştirmişlerdir. Geliştirdikleri sensörün gerinim hassasiyeti $1.878 \mathrm{pm} / \mu \varepsilon$ 'dir. Peng ve diğ. [16], lityum iyon pilin mikro gerinimini izlemek için uygun bir yöntem sağlamak amacıyla $11.49 \mathrm{pm} / \mu \varepsilon$ hassasiyete sahip yeni bir FBG gerinim sensörü geliştirmişlerdir. Araştırmacılar ve firmalar tarafından geliştirilen sensörler ve özellikleri Tablo 1'de özet olarak incelenmiştir. Bu paketleme teknikleri basit yapı, kolay paketleme, rahat kurulum sağlarken taşınımdan dolayı oluşan gerinimi azaltırlar [7].

Tablo 1. FBG Gerinim Sensörleri ve Özellikleri

\begin{tabular}{|c|c|c|}
\hline Sabitleme Eleman1 & Parametre & Değer \\
\hline \multirow{4}{*}{ Ren ve diğ. [7] } & Gerinim hassasiyeti $(\mathrm{pm} / \mu \varepsilon)$ & 2.053 \\
\hline & Ölçüm aralığ $1(\mu \varepsilon)$ & $0-700$ \\
\hline & Boyut (mm) & 15 \\
\hline & Sicaklık telafisi & Hayır \\
\hline \multirow{4}{*}{ Li ve diğ. [10] } & Gerinim hassasiyeti $(\mathrm{pm} / \mu \varepsilon)$ & 2.52 \\
\hline & Ölçüm aralığı $(\mu \varepsilon)$ & $0-200$ \\
\hline & Boyut (mm) & 254 \\
\hline & Sicaklık telafisi & Hayır \\
\hline \multirow{4}{*}{ Zhang ve diğ. [11] } & Gerinim hassasiyeti $(\mathrm{pm} / \mu \varepsilon)$ & 1.814 \\
\hline & Ölçüm aralığ $1(\mu \varepsilon)$ & $-1500-400$ \\
\hline & Boyut $\left(\mathrm{mm}^{3}\right)$ & $300 \times 40 \times 2$ \\
\hline & Sicaklik telafisi & Evet \\
\hline \multirow{4}{*}{ Li ve diğ. [12] } & Gerinim hassasiyeti $(\mathrm{pm} / \mu \varepsilon)$ & 6.2 \\
\hline & Ölçüm aralığ $(\mu \varepsilon)$ & $0-200$ \\
\hline & Boyut $\left(\mathrm{mm}^{3}\right)$ & $36 \times 10.5 \times 1$ \\
\hline & Sicaklık telafisi & Hayır \\
\hline \multirow{4}{*}{ Li ve diğ. [8] } & Gerinim hassasiyeti $(\mathrm{pm} / \mu \varepsilon)$ & 7.72 ve -2.94 \\
\hline & Ölçüm aralığ $1(\mu \varepsilon)$ & $0-225$ \\
\hline & Boyut $\left(\mathrm{mm}^{3}\right)$ & $36 \times 10 \times 1$ \\
\hline & Sicaklık telafisi & Evet \\
\hline \multirow{4}{*}{ Guo ve diğ. [13] } & Gerinim hassasiyeti $(\mathrm{pm} / \mu \varepsilon)$ & 3.357 \\
\hline & Ölçüm aralığı $(\mu \varepsilon)$ & \pm 600 \\
\hline & Boyut (mm) & 100 \\
\hline & Sicaklik telafisi & Hayır \\
\hline
\end{tabular}


Tablo 1. FBG Gerinim Sensörleri ve Özellikleri

\begin{tabular}{|c|c|c|}
\hline \multirow{4}{*}{ Nawrot ve diğ. [14] } & Gerinim hassasiyeti $(\mathrm{pm} / \mu \varepsilon)$ & 31.54 \\
\hline & Ölçüm aralığ $1(\mu \varepsilon)$ & $0-50$ \\
\hline & Boyut $\left(\mathrm{mm}^{3}\right)$ & $380 \times 105 \times 9$ \\
\hline & Sicaklık telafisi & Hayır \\
\hline \multirow{4}{*}{ Yao ve diğ. [15] } & Gerinim hassasiyeti $(\mathrm{pm} / \mu \varepsilon)$ & 1.878 \\
\hline & Ölçüm aralı̆̆ı $(\mu \varepsilon)$ & $0-152$ \\
\hline & Boyut $\left(\mathrm{mm}^{3}\right)$ & $15 \times 12 \times 2$ \\
\hline & Sicaklık telafisi & Evet \\
\hline \multirow{4}{*}{ Peng ve diğ. [16] } & Gerinim hassasiyeti $(\mathrm{pm} / \mu \varepsilon)$ & 11.49 \\
\hline & Ölçüm aralığı $(\mu \varepsilon)$ & \pm 500 \\
\hline & Boyut $\left(\mathrm{mm}^{3}\right)$ & $41 \times 10 \times 1$ \\
\hline & Sicaklık telafisi & Evet \\
\hline \multirow{4}{*}{ HBM-S62WSS [17] } & Gerinim hassasiyeti $(\mathrm{pm} / \mu \varepsilon)$ & 1.2 \\
\hline & Ölçüm aralığ $1(\mu \varepsilon)$ & \pm 5000 \\
\hline & Boyut $\left(\mathrm{mm}^{3}\right)$ & $83 \pm 0.5 \times 23 \pm 0.5 \times 6 \pm 0.5$ \\
\hline & Sicaklık telafisi & Hayır \\
\hline \multirow{4}{*}{ HBM-FS62CSS [17] } & Gerinim hassasiyeti $(\mathrm{pm} / \mu \varepsilon)$ & 1.2 \\
\hline & Ölçüm aralığı $(\mu \varepsilon)$ & \pm 5000 \\
\hline & Boyut $\left(\mathrm{mm}^{3}\right)$ & $130 \pm 0.5 \times 20 \pm 0.5 \times 6 \pm 0.5$ \\
\hline & Sicaklık telafisi & Hayır \\
\hline \multirow{4}{*}{ HBM-FS62PSS [17] } & Gerinim hassasiyeti $(\mathrm{pm} / \mu \varepsilon)$ & 1.2 \\
\hline & Ölçüm aralı̆̆ $1(\mu \varepsilon)$ & \pm 20000 \\
\hline & Boyut $\left(\mathrm{mm}^{3}\right)$ & $40 \pm 0.5 \times 12 \pm 0.5 \times 2 \pm 0.5$ \\
\hline & Sicaklik telafisi & Hayır \\
\hline \multirow{4}{*}{ Micron Optics-os3155 [9] } & Gerinim hassasiyeti $(\mathrm{pm} / \mu \varepsilon)$ & 1.2 \\
\hline & Ölçüm aralığı $(\mu \varepsilon)$ & \pm 2500 \\
\hline & Boyut $\left(\mathrm{mm}^{3}\right)$ & $146 \times 25.4 \times 0.8$ \\
\hline & Sicaklık telafisi & Evet \\
\hline \multirow{4}{*}{ Micron Optics-os3150 [9] } & Gerinim hassasiyeti $(\mathrm{pm} / \mu \varepsilon)$ & 1.4 \\
\hline & Ölçüm aralığı $(\mu \varepsilon)$ & \pm 2500 \\
\hline & Boyut $\left(\mathrm{mm}^{3}\right)$ & $136 \times 25.4 \times 0.8$ \\
\hline & Sicaklık telafisi & Hayır \\
\hline \multirow{4}{*}{ Micron Optics-os3100 [9] } & Gerinim hassasiyeti $(\mathrm{pm} / \mu \varepsilon)$ & 1.4 \\
\hline & Ölçüm aralı̆̆ $1(\mu \varepsilon)$ & \pm 2500 \\
\hline & Boyut $\left(\mathrm{mm}^{3}\right)$ & $36.5 \times 8 \times 0.8$ \\
\hline & Sicaklık telafisi & Hayır \\
\hline
\end{tabular}

\section{FBG Sensöründe Gerinim Transferi}

1991 yılında Nanni ve di $\breve{g}$., optic fiber ve test malzemesi arasında gerinim farklılıklarının olduğunu göstermişlerdir. 1998 yılında da Ansari ve Yuan, optik fiber sensörlerin gerinim transferini araştırmak için bir dizi varsayım önermişlerdir. Son yıllarda da birçok çalışma FBG sensörlerinin gerinim transfer özellikleri üzerine odaklanmıştır. Sınırlı sayıdaki deneysel çalışmalarda, yapıştırıcının elastisite modülü, viskozitesi, kayma modülü (shear modulus), kalınlığı ve uzunluğu gibi parametrelerin ölçüm doğruluğuna etkileri üzerinde durulmuştur [5, 6, 18-20].

$\mathrm{Bu}$ çalışmada, yapıştırıcının kalınlığı ve yapıştırıcının Poisson oranının gerinim transferine etkisi incelenmiştir. Gerinim transfer fonksiyonunu doğrulamak için Torres ve diğ. [21] yaptığ1 çalışma temel alınarak gerinim sensörünün göreceli hatasının bir ölçüsü olan $\Delta \varepsilon(\%)$ Denklem $\Delta \varepsilon(\%)=\frac{\left(\varepsilon_{t}-\varepsilon_{f}\right)}{\varepsilon_{t}} 100$

(1)'deki 
gibi hesaplanmıştır. Denklemde gösterilen $\varepsilon_{t}$ ve $\varepsilon_{f}$ sırasıyla test malzemesinin ve optik fiberin yüzeylerindeki deformasyonları temsil eder. $\Delta \varepsilon(\%)$ değerinin sıfıra yakın olması test malzemesinden optik fibere neredeyse mükemmel bir gerinim transferinin olduğunu göstermektedir [21].

$$
\Delta \varepsilon(\%)=\frac{\left(\varepsilon_{t}-\varepsilon_{f}\right)}{\varepsilon_{t}} 100
$$

\section{$\mathrm{Bu}$}

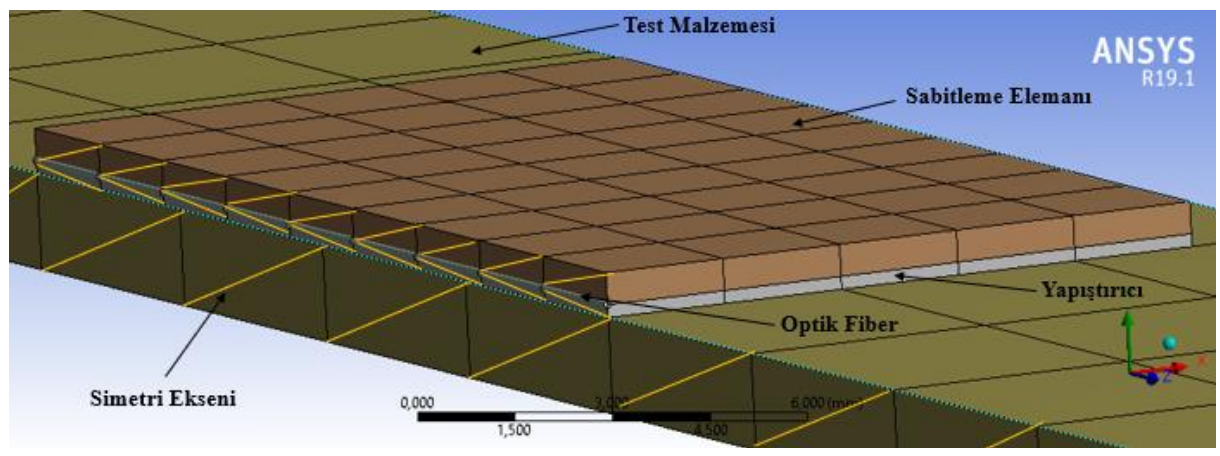

Şekil

Tablo

\begin{tabular}{ccc}
\hline Yap1 & Elastisite & Poisson \\
\hline FBG & 74.52 & 0.17 \\
\hline Yap1ştırıc1 & 13 & 0.2 \\
\hline Sabitleme & 11 & 0.4 \\
\hline Test & 200 & 0.3 \\
\hline
\end{tabular}

Elde

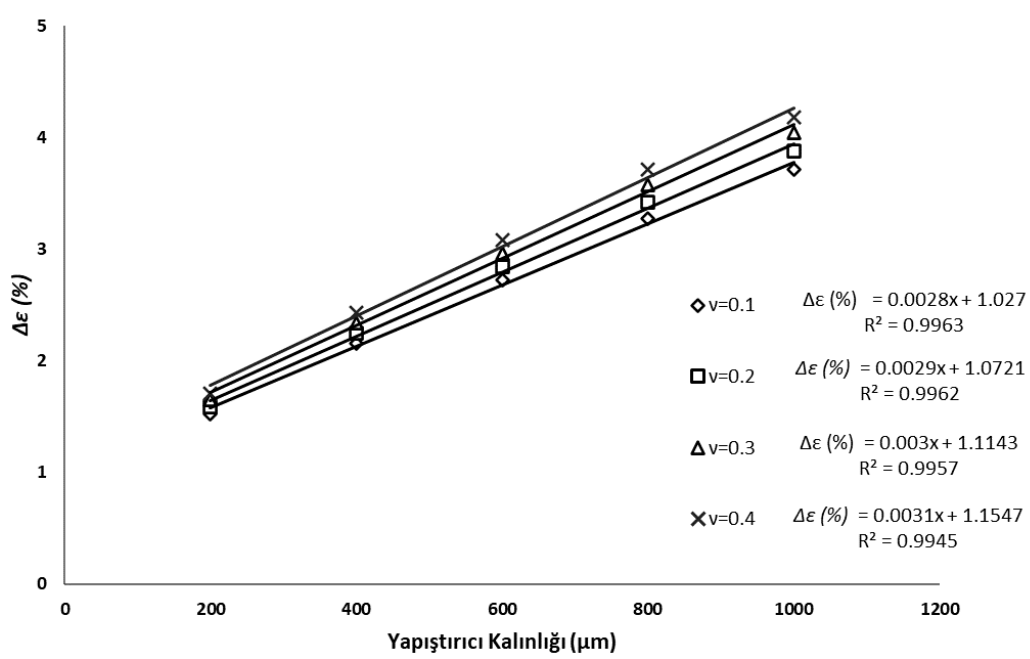

Şekil 
4.

Yapisal

$\mathrm{Bu}$

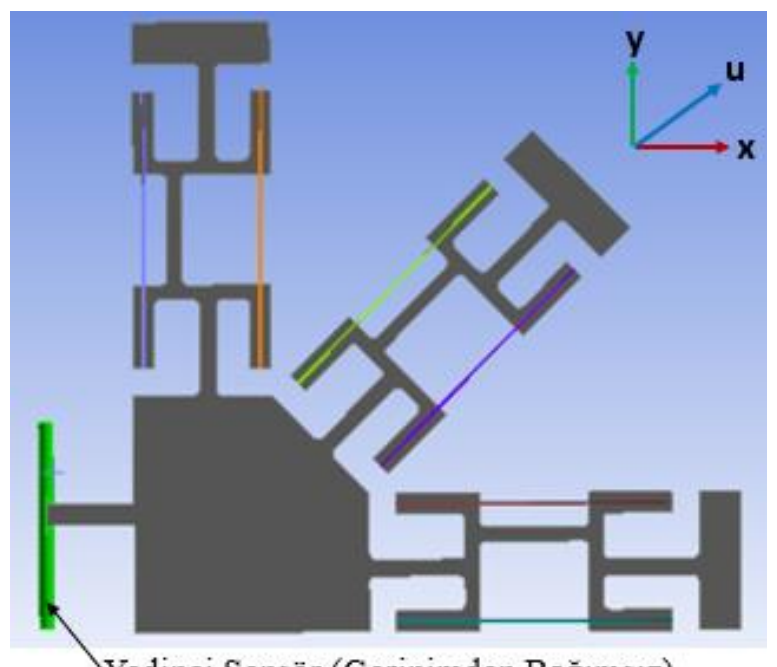

Yedinci Sensör (Gerinimden Bağımsız)
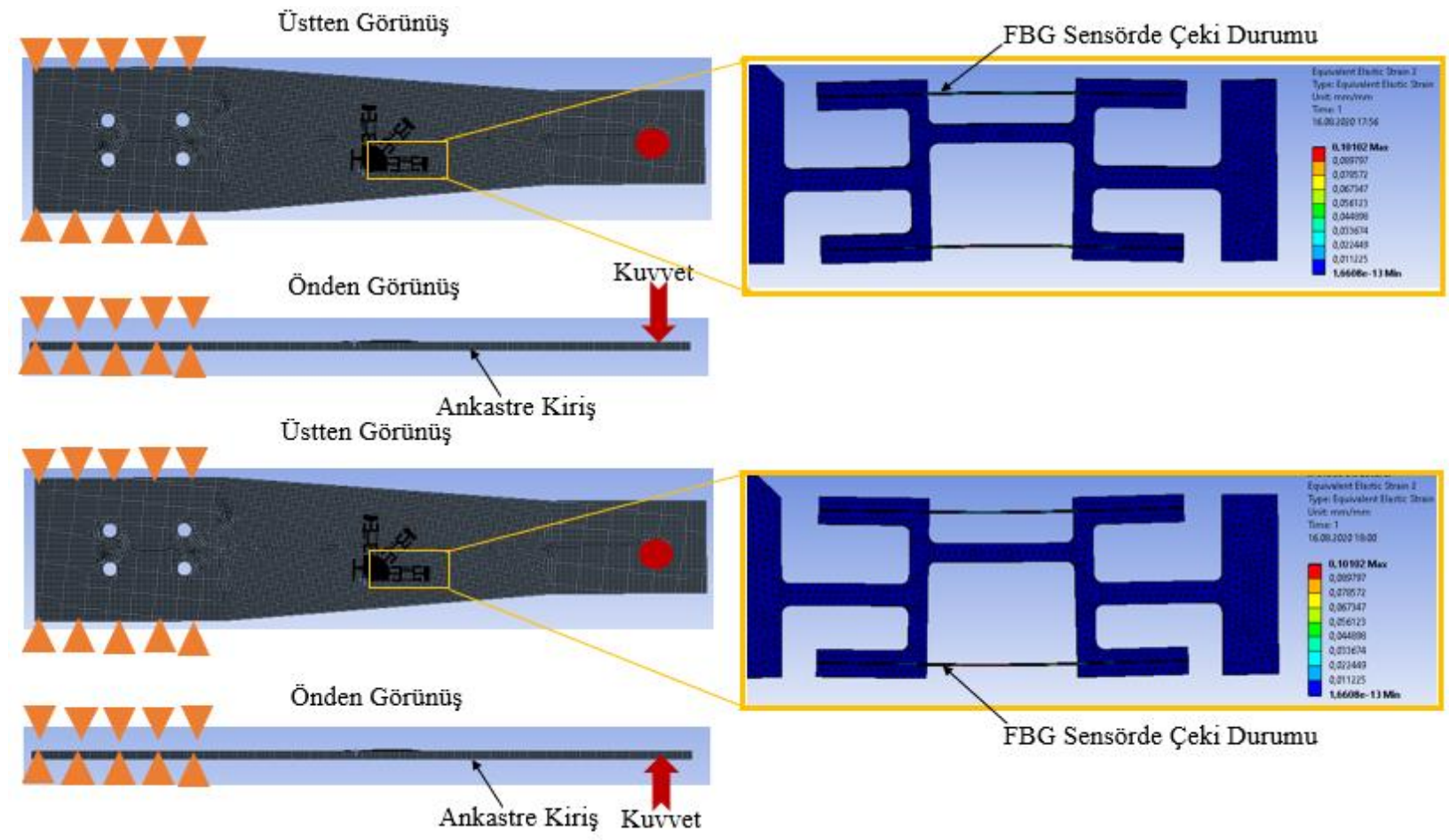

Ankastre Kiriş Kuvvet

Şekil

Tablo

\begin{tabular}{cccc}
\hline Yap1 & Basit & Elastisite & Poisson \\
\hline FBG & Radyus: & 72 & 0.17 \\
\hline
\end{tabular}




\begin{tabular}{|c|c|c|c|}
\hline Yapıştırıc1 & Kalınlık: & 4 & 0.38 \\
\hline Sabitleme & Kalınlık: & 193 & 0.27 \\
\hline Test & Kalınlık: & 200 & 0.30 \\
\hline
\end{tabular}

Ana gerinim ile x-ekseni arasındaki açı $\alpha_{0}$ 'dır. İki boyutlu gerinimin değeri ve yönü Denklem $\operatorname{cmin}=\frac{1}{2}\left[\left(\varepsilon_{x}+\varepsilon_{y}\right)-\sqrt{\left(\varepsilon_{x}-\varepsilon_{u}\right)^{2}+\left(\varepsilon_{u}-\varepsilon_{y}\right)^{2}}\right]$

2), Denklem smax $=\frac{1}{2}\left[\left(\varepsilon_{x}+\varepsilon_{y}\right)+\sqrt{\left(\varepsilon_{x}-\varepsilon_{u}\right)^{2}+\left(\varepsilon_{u}-\varepsilon_{y}\right)^{2}}\right]$

3

$$
\begin{aligned}
& \mathrm{E} \\
& F_{\text {min }}=\frac{1}{2}\left[\left(\varepsilon_{x}+\varepsilon_{y}\right)-\sqrt{\left(\varepsilon_{x}-\varepsilon_{u}\right)^{2}+\left(\varepsilon_{u}-\varepsilon_{y}\right)^{2}}\right] \\
& \bar{R}_{\text {max }}=\frac{1}{2}\left[\left(\varepsilon_{x}+\varepsilon_{y}\right)+\sqrt{\left(\varepsilon_{x}-\varepsilon_{u}\right)^{2}+\left(\varepsilon_{u}-\varepsilon_{y}\right)^{2}}\right] \\
& \mathrm{e} \\
& \underset{4}{\tan 2} \alpha_{0}=\frac{2 \varepsilon_{u}-\varepsilon_{x}-\varepsilon_{y}}{\varepsilon_{x}-\varepsilon_{y}} \\
& 8
\end{aligned}
$$

\section{Sonuç}

4

4 FBG sensörler için tasarlanan sabitleme elemanları farklı sektörlerde birçok uygulamada łullanılmaktadır. Tasarımına bağlı olarak sağladığı avantajlar ve dezavantajlar değerlendirilerek 9ygulama için en uygun olan sabitleme elemanının seçilmesi oldukça önemlidir.

$1 \quad \mathrm{Bu}$ bildiride, dalga boyu tabanlı bir sensör olan FBG sensörlerin avantajları, yüzeye bağlantı yöntemleri, akademik çalışmalar sonucu ve endüstriyel olarak geliştirilen sabitleyici ęleman çeşitleri incelenmiştir. Bununla birlikte, FBG sensör uygulamalarında gerinim transferi incelenerek yapıştırıcı kalınlığının ve Poisson oranının ölçüm doğruluğuna etkileri üzerinde durulmuştur. İki boyutlu gerinim ölçümü için yeni bir FBG rozet sabitleyici eleman tasarımı yapılmıştır.

1

\section{Referanslar}

M] Günaydın A.C., Hatipoğlu A., Fidanboylu K. Havacılık Sektöründe Fiber Optik E Uygulamaları. TMMOB Makina Mühendisleri Odası X. Ulusal Uçak, Havacılık ve Uzay R Mühendisliği Kurultayı. Eskişehir. 2019.

[e] Fidanboylu K., Efendioğlu H.S. Fiber Optic Sensors and Their Applications. $5^{\text {th }}$ International E Advanced Technologies Symposium (IATS'09). May 13-15, 2009. Karabuk, Turkey.

F3] Yücel M., Öztürk N.F., Torun M. Fiber Bragg Izgara Sensör Dizisi Tabanlı Sıcaklık Ölçüm O Sistemi Tasarımı ve Uygulaması. Journal of the Faculty of Engineering and Architecture of R Gazi University 32:3 (2017) 957-964.

A] Pachava V.R., Kamineni S., Madhuvarasu S.S., Putha K. A High Sensitive FBG Pressure A Sensor Using Thin Metal Diaphragm. J Opt (April-June 2014) 43(2):117-121.

[5] Jiang S. Research on the Strain Transfer Mechanism of FBG Radian Strain Sensor. 
International Journal of Optic and Photonic Engineering, 3:013.

[6] J. Zhou, Z. Zhou, D. Zhang. Study on Strain Transfer Characteristics of Fiber Bragg Grating Sensors. Journal of Intelligent Material Systems and Structures, Vol. 21, 1117.

[7] Ren L., Chen J., Li H.-N., Song G., Ji X. Design and Application of a Fiber Bragg Grating Strain Sensor with Enhanced Sensitivity in the Small-Scale Dam Model. Smart Mater. Struct. 18 (2009) 035015 (7pp).

[8] Li R., Tan Y., Chen Y., Hong L. Zhou Z. Investigation of Sensitivity Enhancing and Temperature Compensation for Fiber Bragg Grating (FBG)-Based Strain Sensor. Optical Fiber Technology 48 (2019) 199-206.

[9] http://www.micronoptics.com/products/sensing-solutions/sensors/ (14.07.2020)

[10] Li L., Zhang D., Liu H., Guo Y., Zhu F. Design of an Enhanced Sensitivity FBG Strain Sensor and Application in Highway Bridge Engineering. Photonic Sensors / Vol. 4, No. 2, 2014: $162-167$.

[11] Zhang L., Liu Y., Gao X., Xia Z. High Temperature Strain Sensor Based on a Fiber Bragg Grating and Rhombus Metal Structure. Vol. 54, No. 28 / October 12015 / Applied Optics.

[12] Li R., Chen Y., Tan Y., Zhou Z., Li T., Mao J. Sensitivity Enhancement of FBG-Based Strain Sensor. Sensors 18 (5) (2018) 1607.

[13] Guo Y., Kong J., Liu H., Hu D., Qin L. Design and Investigation of a Reusable SurfaceMounted Optical Fiber Bragg Grating Strain Sensor. IEEE Sensors Journal, Vol. 16, No. 23, December 1, 2016.

[14] Nawrot U., Geernaert T., Pauw B.D., Anastasopoulos D., Reynders E., Roeck G.D., et al. Development of a Mechanical Strain Amplifying Transducer with Bragg Grating Sensor for Low-Amplitude Strain Sensing. Smart Mater. Struct. 26 (2017) 075006 (10pp).

[15] Yao K., Lin Q., Jiang Z., Zhao N., Peng G.D., Tian B., et al. Design and Analysis of a Combined Strain-Vibration-Temperature Sensor with Two Fiber Bragg Gratings and a Trapezoidal Beam. Sensors 2019, 19, 3571.

[16] Peng J., Jia S., Jin Y., Xu S., Xu Z. Design and Investigation of a Sensitivity-Enhanced Fiber Bragg Grating Sensor for Micro-Strain Measurement. Sensors and Actuators A 285 (2019) $437-447$.

[17] https://www.hbm.com/en/4599/new-light-optical-fiber-sensors/ (14.07.2020)

[18] Zhang Y., Zhu L., Luo F., Dong M., Yang R., He W., et al. Comparison of Metal-Packaged and Adhesive-Packaged Fiber Bragg Grating Sensors. IEEE Sensors Journal, Vol. 16, No. 15, August 1, 2016.

[19] Tian H., Liu D.G., Wang Y.P., Wang Q.L. Effect of Adhesive Type on the Sensitivity Coefficient of FBG Sensor Bonded on the Surface of CFRP. Optoelectronics Lett. Vol.15 No.4.

[20] Castro-Caicedo A.J., Torres P., Nieto-Callejas M.J., Lain-Huerta R., Suarez-Burgoa L.O. Manufacturing and Reliability of a Fiber Bragg Grating Strain Sensor Designed for Uniaxial Compression Test of Rocks. DYNA, 86(208), 234-242.

[21] Torres B., Paya-Zaforteza I., Calderon P.A., Adam J.M. Analysis of the Strain Transfer in a New FBG Sensor for Structural Health Monitoring. Engineering Structures 33 (2011) 539548.

[22] Guo Y., Zhu X., Ni Y. Temperature Compensated Three-Directional Fiber Bragg Grating Strain Sensor with Big Measurement Range and High Accuracy. Opt. Eng. 57(11), 117113 (2018). 
[23] Ramos C.A., Oliveira R., Marques A.T., Frazao O. Design and Experimental Evaluation of a Composite Strain Rosette Using Fiber Bragg Grating. Microwave and Optical Technology Letters / Vol. 53, No. 8, August 2011.

[24] Shen W., Yan R., Xu L., Tang G., Chen X. Application Study on FBG Sensor Applied to Hull Structural Health Monitoring. Optik 126 (2015) 1499-1504. 